EPJ Web of Conferences 59, 03015 (2013)

DOI: $10.1051 /$ epjconf/20135903015

(C) Owned by the authors, published by EDP Sciences, 2013

\title{
Particle size effect on velocity of gold particle embedded laser driven plastic targets
}

\author{
L.J. Dhareshwar ${ }^{1, a}$, S. Chaurasia ${ }^{2}$, K. Manmohan ${ }^{3}$, J. Badziak ${ }^{4}$, J. Wolowski ${ }^{4}$, \\ A. Kasperczuk ${ }^{4}$, T. Pisarczyk ${ }^{4}$, L. Ryc ${ }^{4}$, M. Rosinski ${ }^{4}$, P. Parys ${ }^{4}$, P. Pisarczyk ${ }^{4}$, \\ J. Ullschmidt ${ }^{5}$, E. Krousky ${ }^{5}$ and K. Masek ${ }^{5}$
}

${ }^{1}$ Raja Ramanna Fellow, Physics Group, Bhabha Atomic Research Centre, Mumbai, India

${ }^{2}$ High Pressure Physics and Synchrotron Radiation Division, Bhabha Atomic Research

Centre

${ }^{3}$ Radiation and Photochemistry Division, Bhabha Atomic Research Centre

${ }^{4}$ Institute of Plasma Physics and Laser Microfusion, Warsaw, Poland

${ }^{5}$ PALS Research Centre, ASCR, Czech Republic

\begin{abstract}
A scheme to enhance the target foil velocity has been investigated for a direct drive inertial fusion target. Polymer PVA (polyvinyl alcohol or $\left.\left(\mathrm{C}_{2} \mathrm{H}_{4} \mathrm{O}\right)_{\mathrm{n}}\right)$ target foils of thickness $15-20 \mu \mathrm{m}$ were used in plain form and also embedded with gold in the nano-particle (Au-np) or micro-particle (Au-mp) form. Nanoparticles were of $20-50 \mathrm{~nm}$ and micro-particles of $2-3 \mu \mathrm{m}$ in size. $17 \%$ higher target velocity was measured for foils embedded with nano-particle gold (Au-np) as compared to targets embedded with micro-particles gold (Au-mp). The weight of gold in both cases was in the range $40-55 \%$ of the full target weight (atomic percentage of about 22\%). Experiments were performed with the single beam of the Prague Asterix Laser System (PALS) at $0.43 \mu \mathrm{m}$ wavelength ( $3 \omega$ of the fundamental wavelength), 120 Joule energy and $300 \mathrm{psec}$ pulse duration. Laser intensity on the target was about $10^{15} \mathrm{~W} / \mathrm{cm}^{2}$. A simple model has been proposed to explain the experimental results.
\end{abstract}

\section{INTRODUCTION}

Several target schemes have evolved over the past years to enhance laser to target coupling efficiency together with a stable ablative acceleration of targets in direct-drive Inertial Confinement Fusion (ICF) scheme using high gain pellet targets [1]. Results on stable and enhanced acceleration in plastic foil targets doped with $20 \%$ by weight of tungsten and ablated by laser pulses of several nanoseconds has been earlier reported by Dhareshwar and Pant [2]. Fujioka et al. [3] have proposed a reduction of the RT instability growth rate by the so-called double plasma ablation, where, in addition to the ablation surface formed due to electron conduction induced by laser light, a new ablation surface is created by X-ray radiation from high- $Z$ atoms doped to the low- $Z$ (plastic) material of the target. Such target structure can lead to higher ablation velocity and longer density scale length and, as a result, to the damping of higher RT modes in a planar case. This idea has been proved both numerically and experimentally in $20-\mu \mathrm{m}$ thick plastic targets doped with $3 \%$ bromine. The targets were observed to be accelerated to the velocity of $6 \times 10^{7} \mathrm{~cm} / \mathrm{s}$ when irradiated by a $0.35-\mu \mathrm{m}, 2.5-\mathrm{ns}$ laser pulse of intensity $\sim 10^{14} \mathrm{~W} / \mathrm{cm}^{2}$.

We performed experiments [4] with $20 \mu \mathrm{m}$ thick cellulose acetate targets doped with bromine at laser intensity of $10^{15} \mathrm{~W} / \mathrm{cm}^{2}$, using the PALS laser, Prague, where the ablated doped targets were found to

\footnotetext{
ae-mail: dharesh@barc.gov.in
}

This is an Open Access article distributed under the terms of the Creative Commons Attribution License 2.0, which permits unrestricted use, distribution, and reproduction in any medium, provided the original work is properly cited. 


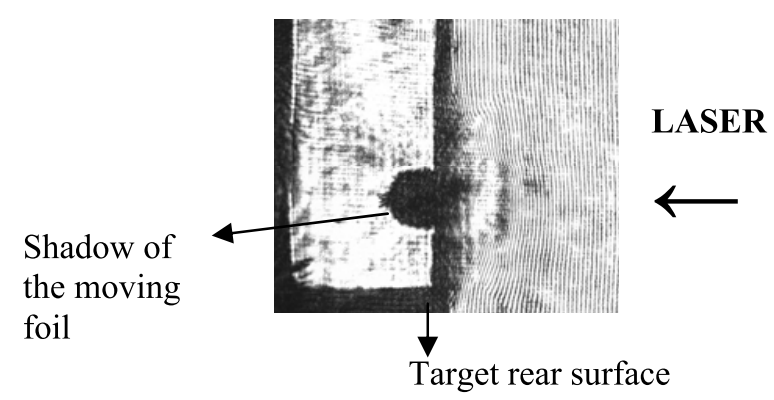

Figure 1. Typical interferogram cum shadowgram recorded for the target foil, with the three-frame interferometer at a $8 \mathrm{~ns}$ probe pulse delay.

have a more collimated plasma flow with higher density and velocity (when comparing with results from Ref. [2]). Furthering this argument, a still larger enhancement in target velocity can be expected when targets are doped with an increased concentration of a material with a higher atomic number.

\section{EXPERIMENTS}

A single beam of the PALS laser of $120 \mathrm{~J} / 300 \mathrm{psec}(0.434 \mu \mathrm{m}$ wavelength $)$ was incident normal on the target foil. Laser intensity was about $10^{15} \mathrm{~W} / \mathrm{cm}^{2}$. Plasma density and velocity of the target foil were measured with a three-frame optical interferometer using a $3 \omega$ probe beams having $2 \mathrm{~ns}, 5 \mathrm{~ns}$ and $8 \mathrm{~ns}$ delay with respect to the main laser beam. A CCD camera with digital data acquisition system supported by requisite software could yield 3-D plasma density profiles as well as shadowgrams of the accelerated foil target. Semiconductor diodes with different X-ray filters (6-20 $\mu \mathrm{m}$ aluminum foils) were used to record X-ray emission in the range $1.6-20 \mathrm{keV}$. An X-ray streak camera with a temporal resolution of 5 ps was placed vertically above the moving foil to record the temporal variation of $x$-ray flux.

The PVA foil targets were made to have 1-2 $\mu \mathrm{m}$ Au particles (Au-mp) or 15-20 nm Au particles (Au-np) uniformly dispersed. Weight of the gold particles (Au-np as well as Au-mp) was about 55\% of PVA weight in the target (22\% atomic percentage). The density of the pure un-doped PVA foil, the one doped with Au nano-particles, and the one doped with Au micro-particles were $0.997 \mathrm{~g} / \mathrm{cm}^{3}, 1.64 \mathrm{~g} / \mathrm{cm}^{3}$ and $1.72 \mathrm{~g} / \mathrm{cm}^{3}$, respectively.

\section{RESULTS AND DISCUSSION}

The typical interferogram cum shadowgram picture recorded at a probe delay of $8 \mathrm{~ns}$ for the accelerated foil target is shown in Fig. 1 Target foil velocity is measured by plotting the target foil movement at the different delays as shown in Fig. 2.

It is clearly observed that the PVA targets containing Au-np exhibit a higher velocity compared to pure PVA targets and PVA with Au-mp. Fig. 3(a)-(c) show the plasma density distribution in all three targets. A well collimated, higher plasma density distribution is seen in the case of PVA with Au-np, as compared with other two. X-ray streak pictures seen in Fig. 4 clearly show a higher X-ray intensity in the case of Au-np PVA. The integrated X-ray yield in Fig. 5 exhibits more than twice as much enhancement of X-ray emission from Au-np PVA targets while compared to Au-mp PVA targets. Modeling of the interaction of an intense laser field with a dielectric containing metallic nano-particles has been done by several authors [5]. This was done to study laser damage in laser glasses containing nano-scale metal inclusions. We have evolved a model based on the initial electron kinetics and emission from metal particles exposed to intense laser radiation which modifies the absorption of the dielectric in which they are embedded. 


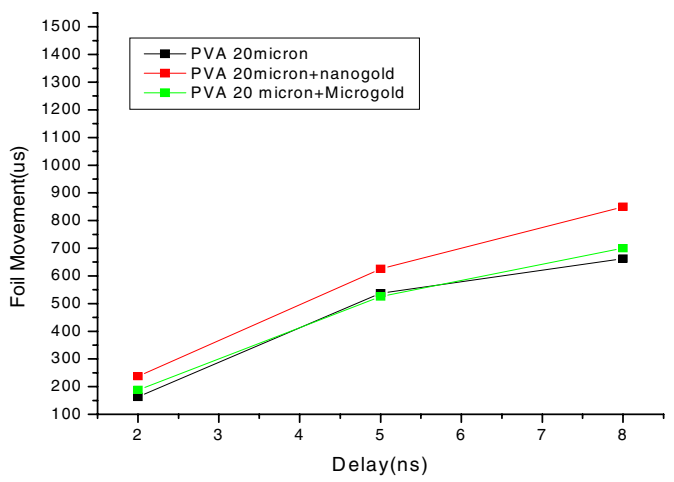

Figure 2. Target foil motion versus delay.

(a)
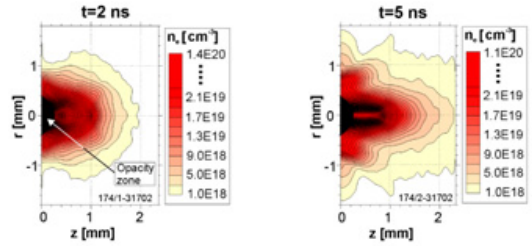

$\mathrm{t}=\mathbf{2} \mathrm{ns}$

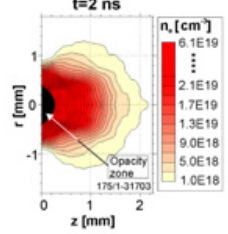

(b)

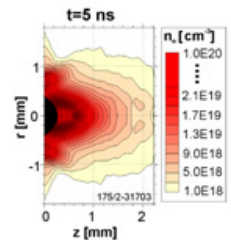

$\mathrm{t}=2 \mathrm{~ns}$

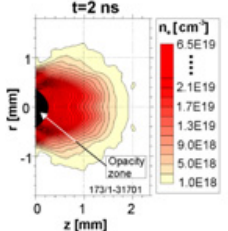

(c)

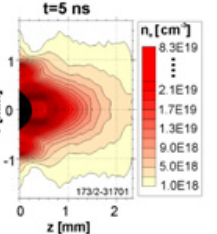

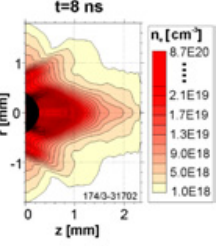
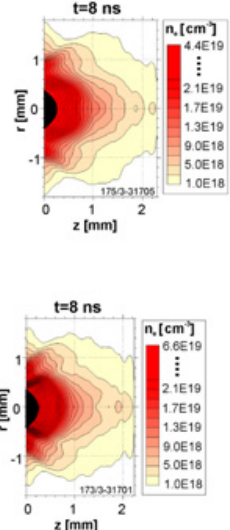

Figure 3. Isodensity plots and plasma density distribution for (a) PVA with Au-np, (b) PVA with Au-mp, and (c) un-doped PVA foil targets.
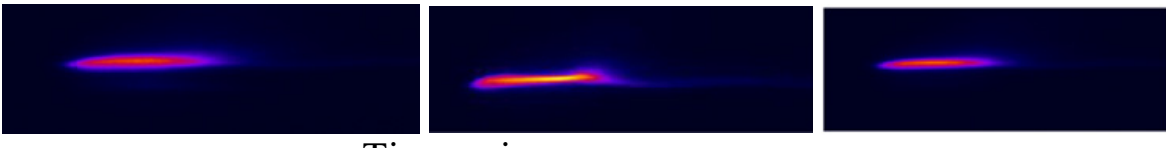

Time axis

Figure 4. X-ray streak pictures of plasma emission from pure PVA, PVA with Au-np, PVA with Au-mp, left to right.

A metal particle embedded in the PVA dielectric acts as a "short cut" between the valence and conduction bands of PVA.

The role of nano-particles has been ascribed to ejecting a large number of electrons into the dielectric causing a strongly absorbing plasma around the particles. Then, the conduction electrons in PVA absorb 


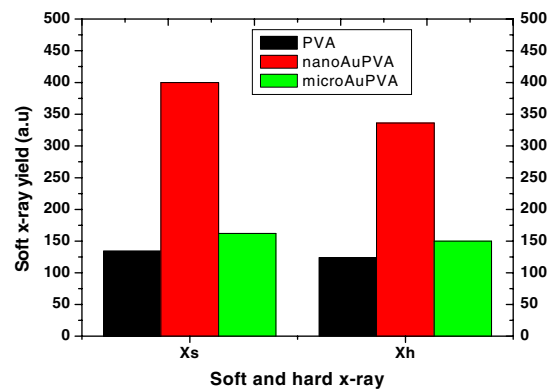

Figure 5. Soft and hard x-ray ray yield for PVA and gold doped PVA targets.

the laser energy through the electron-photon-phonon collisions, leading to energy deposition much higher than in the absence of the nano-particles. Further, the nano-particles heated by the laser become intense source of X-rays which are absorbed by the surrounding PVA matrix, leading to an overall higher energy deposition in the target [6]. The nano-particle electron heating and kinetics under a strong laser excitation field has been modeled in the simplest manner by calculating the absorption by the nano-particles using Mie's theory. The number of ejected electrons from the metal nano-particles by the thermionic and photoelectric effects has been calculated as a function of the particle's dimension and laser pulse duration as well as intensity.

The metal nano-particle essentially consists of an electron system and phonon system, each in local equilibrium. Therefore, it needs a two-temperature treatment using the electron temperature $T_{e}$ and the lattice temperature $T_{l}$. An equation concerning the evolution of temperature around a nano-particle is described by the PVA temperature $T_{p v a}$. The rate of energy loss is observed to increase with decrease in particle size. The exchange of energy between the nano-particle and PVA matrix takes place by the mechanism of diffusion. The diffusion time $\tau$ is very small compared to the laser pulse duration. Characteristic time of energy exchange between the nano-particle and PVA matrix can be defined as- $\tau_{m}=a^{2} C_{p v a} / 3 \kappa \approx 25 a^{2}(\mathrm{~nm})^{2}$ fs. Thus, for a $20 \mathrm{~nm}$ particle size, $\tau_{m}=10 \mathrm{ps}$. For micro-particles, the situation is different, for a 2-3 micron size $\tau_{m}=0.1 \mu \mathrm{s}$. In PVA the micro particles are seen to agglomerate and this can further increase $\tau_{m}$.

In conclusion, if the same weight of gold is dispersed within the PVA target, the overall laser absorption of target is enhanced when the gold is in nano-particle form. This leads to an increase in target velocity and therefore kinetic energy of the accelerated target. Thus, for identical laser energy, the PVA targets with Au-np would be having an enhanced hydrodynamic efficiency.

\section{References}

[1] L. Phillips, J.H. Gardner, S.E. Bodner et al., Laser and Part. Beams 17, 225 (1999)

[2] L.J. Dhareshwar, H.C. Pant, Proc. Plasma Phys. Contr. Fus. 3, 165 (1992)

[3] S. Fujioka, A. Sunhara, K. Nishihara et al., Phys. Rev. Lett. 92, 195001 (2004)

[4] J. Badziak, A. Kasperczuk, P. Parys et al., Appl. Phys. Lett. 92, 211502 (2008)

[5] V.K. Pustovalov, Chem. Phys. 308, 103 (2005)

[6] A. Kaiser, B. Rethfeld, M. Vicanek, G. Simon, Phys. Rev. B 61, 11437 (2000); P. Grua et al., Phys. Rev. B 68, 035424 (2003) 